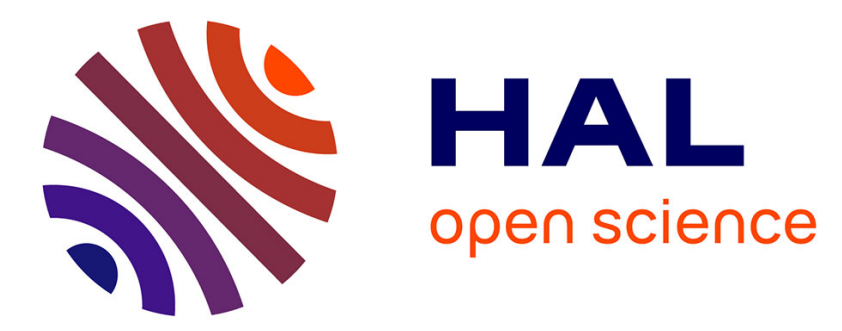

\title{
Is the Taylor-Proudman theorem exact in unbounded domains? Case study of the three-dimensional stability of a vortex pair in a rapidly rotating fluid Paul Billant
}

\section{- To cite this version:}

Paul Billant. Is the Taylor-Proudman theorem exact in unbounded domains? Case study of the three-dimensional stability of a vortex pair in a rapidly rotating fluid. Journal of Fluid Mechanics, 2021, 920, 10.1017/jfm.2021.431 . insu-03329722

HAL Id: insu-03329722

https://hal-insu.archives-ouvertes.fr/insu-03329722

Submitted on 31 Aug 2021

HAL is a multi-disciplinary open access archive for the deposit and dissemination of scientific research documents, whether they are published or not. The documents may come from teaching and research institutions in France or abroad, or from public or private research centers.
L'archive ouverte pluridisciplinaire $\mathbf{H A L}$, est destinée au dépôt et à la diffusion de documents scientifiques de niveau recherche, publiés ou non, émanant des établissements d'enseignement et de recherche français ou étrangers, des laboratoires publics ou privés. 


\title{
Is the Taylor-Proudman theorem exact in unbounded domains?: case study of the three-dimensional stability of a vortex pair in a rapidly rotating fluid
}

\author{
Paul Billant $\dagger$ \\ Laboratoire d'Hydrodynamique (LadHyX), CNRS, Ecole Polytechnique, Institut Polytechnique de Paris, \\ 91120 Palaiseau, France
}

(Received $\mathrm{xx}$; revised $\mathrm{xx}$; accepted $\mathrm{xx}$ )

Owing to the Taylor-Proudman theorem, it is generally believed that rotating flows should have a two-dimensional dynamic for rapid background rotation. Yet, we show that two infinitely long counter-rotating vertical vortices remain always unstable to three-dimensional perturbations even for large rotation rate about the vertical axis. The dominant instability consists in quasiantisymmetric displacements of the two vortices. Its growth rate is independent of the rotation rate when it is sufficiently large while the most amplified vertical wavelength scales like the rotation rate. Direct numerical simulations show that the instability leads ultimately to a full three-dimensional breakdown of the vortex pair.

In other words, a two-dimensional vortex pair will develop spontaneously three-dimensional variations even in the limit of infinite rotation rate but the wavelength will tend to infinity. This implies that the Taylor-Proudman theorem can be strictly valid only in vertically bounded flows.

The scaling for the wavelength is next generalized by showing that the typical vertical scale in rapidly rotating unbounded flows is $L_{v} \sim L_{h} /$ Ro where $L_{h}$ is the horizontal scale and $R o$ the Rossby number. This scaling law is shown to derive from a self-similarity of the Navier-Stokes equations for $R o \ll 1$. The resulting reduced equations are identical to those obtained first by Julien et al. (1998) and Nazarenko \& Schekochihin (2011) by means of multiscale analyses in the cases of rotating convection or turbulence.The self-similarity demonstrated herein suggests that these reduced equations are valid for any rapidly rotating unbounded flow.

\section{Introduction}

The well-known Taylor-Proudman theorem states that rapidly rotating flows should be uniform along the axis of rotation (here the vertical axis) (Greenspan 1968; Davidson 2013). This theorem can be derived easily by assuming an inviscid, incompressible and unbounded fluid and by considering the asymptotic limit of an infinitely rapid rotation compared to the timescale of fluid motions.

Although the tendency towards vertically coherent flows is well observed, many experiments and numerical simulations show that rapidly rotating flows are rarely purely two-dimensional (see Julien et al. 1998; Sprague et al. 2006; Moisy et al. 2011; Davidson 2013; Godeferd \& Moisy 2015; Deusebio et al. 2014; van Kan \& Alexakis 2020, and references therein). This may originate from a violation of one assumption behind the Taylor-Proudman theorem, for example: if the Rossby number is not sufficiently small, if boundary layers are present or if there are thermal effects like in rotating convection.

However, when these assumptions are fulfilled, the Taylor-Proudman theorem is generally

$\dagger$ Email address for correspondence: billant@ladhyx.polytechnique.fr 
interpreted as a proof that vertical variations can not arise spontaneously in rapidly rotating flows. In particular, the consensus is that three-dimensional instabilities are precluded in the limit of large rotation rate. The elliptic instability is indeed commonly accepted to be stabilized for rapid rotation even if, paradoxically, the theoretical expression for its growth rate is non-zero for infinite rotation rate. One reason given is that the unstable vertical wavenumber band shrinks and tends to zero for infinite rotation (Potylitsin \& Peltier 1999; Sipp et al. 1999; Le Dizes 2000; Godeferd et al. 2001). The case of the centrifugal instability is simpler since it is well established that this instability is suppressed for large rotation rate (Kloosterziel \& van Heijst 1991; Sipp \& Jacquin 2000). More generaly, Gallet (2015) and Seshasayanan \& Gallet (2020) have proved rigorously that two-dimensional turbulent flows bounded along the vertical direction are stable to three-dimensional perturbations when the rotation is high enough.

However, when the flow is unbounded, some authors have argued that an exact twodimensionalisation can not occur even for infinite rotation rate because there always exist three-dimensional inertial waves with the same timescale as advective motions so that they can couple (Cambon et al. 2004; Nazarenko \& Schekochihin 2011; Deusebio et al. 2014; Alexakis \& Biferale 2018; van Kan \& Alexakis 2020).

In this context, we study in $\S 2$ the linear stability of two counter-rotating vortices whose axes are aligned with the background rotation vector. Strikingly, we show that this two-dimensional base flow remains unstable to three-dimensional perturbations when the rotation is rapid. The growth rate of the dominant instability becomes indeed independent of the rotation rate when it is sufficiently large while the dominant wavelength increases proportionally to the rotation rate. Some Direct Numerical Simulations of the dominant instability are further presented in $\S 3$.

In $\S 4$, we next generalize the scaling law for the vertical wavelength by performing a scaling analysis of the Navier-Stokes equations for large rotation rate in the case of an unbounded flow. The results are summarized and discussed in $\$ 5$.

\section{Three-dimensional stability of a counter-rotating vortex pair in a rotating fluid}

In this section, the three-dimensional stability of a counter-rotating vertical vortex pair in a rotating fluid is analyzed as a function of the rotation rate, starting from the well-known nonrotating limit (Leweke et al. 2016). The effects of the viscosity and the separation distance between the two vortices will be also investigated.

\subsection{Methods}

We use the Navier-Stokes equations for an incompressible fluid with constant density $\rho_{0}$ in a frame rotating about the vertical axis $\boldsymbol{e}_{z}$ at rate $\Omega_{b}$ :

$$
\begin{gathered}
\partial_{t} \boldsymbol{u}+\boldsymbol{u} \cdot \boldsymbol{\nabla} \boldsymbol{u}+2 \Omega_{b} \boldsymbol{e}_{z} \times \boldsymbol{u}=-\rho_{0}{ }^{-1} \nabla p+v \Delta \boldsymbol{u}, \\
\boldsymbol{\nabla} \cdot \boldsymbol{u}=0,
\end{gathered}
$$

where $\boldsymbol{u}=\left(u_{x}, u_{y}, u_{z}\right)$ is the velocity, $p$ the pressure, $v$ the viscosity and $(x, y, z)$ are the Cartesian coordinates.

To perform the stability analysis, a base flow consisting in a quasi-steady vortex pair has been first computed by integrating the two-dimensional Navier-Stokes equations initialized by two counter-rotating Lamb-Oseen vortices with circulations $\pm \Gamma$ and radius $R_{0}$, separated by a distance $b$ along the $y$ direction. In the following, the length and time units will be taken as $R_{0}$ and $2 \pi R_{0}^{2} / \Gamma$, respectively. The numerical code uses a pseudo-spectral method with periodic boundary conditions (Deloncle et al. 2008). Most of the aliasing errors are eliminated by truncating $95 \%$ of the modes along each direction. Time advancement is carried out with the fourth-order Runge Kutta scheme for the nonlinear term and exact integration for the viscous and diffusive terms. 


$$
\begin{array}{c||ccc|ccc}
\frac{L_{x} \times L_{y}}{N_{x} \times N_{y}} \| \frac{60 \times 32}{512 \times 320} & \frac{120 \times 64}{1024 \times 640} & \frac{240 \times 128}{2048 \times 1280} & \frac{120 \times 64}{512 \times 320} & \frac{120 \times 64}{1024 \times 640} & \frac{120 \times 64}{2048 \times 1280} \\
\hline \sigma 2 \pi b^{2} / \Gamma \| 0.4911089 & 0.4863921 & 0.4863124 & 0.4862474 & 0.4863921 & 0.4863918
\end{array}
$$

TABLE 1. Non-dimensional growth rate $\sigma 2 \pi b^{2} / \Gamma$ when the domain sizes are varied keeping the mesh sizes fixed (first three columns) and when the mesh sizes are varied while keeping the box sizes constant (fourth to sixth columns) for $b=4, R e=500, R o=0.8$ and $k R=0.00448$.

The time step is $\delta t=0.02$. The horizontal size of the computational domain is chosen sufficiently large: $L_{x}=120, L_{y}=60+b$ in order to minimize the effects of the periodic boundary conditions. Such large non-squared domain is necessary because the eigenmodes exhibit wide wave trains in the $x$ direction for large rotation rate. The resolution used is $1024 \times 640$. The Reynolds number and the separation distance have been always set to $R e=\Gamma /(2 \pi v)=500$ and $b=4$, except in $\$ 2.2 .2$ when studying the effect of $b$ and viscous effects.

At time evolves, the two vortices quickly adapt to each other and reach a quasi-stationnary state in which the vortex pair propagates at constant speed (Sipp et al. 2000). Hence, the flow is quasi-steady in the reference frame traveling at that speed. The base state used for the following three-dimensional stability analysis has been taken as the flow at time $t=30$ (figure 1a). The characteristics of the vortices at that time have been obtained by fitting the vorticity field by the least-square method to two Lamb-Oseen vortices with circulation $\Gamma$ and separation distance $b$. For example, for $b=4$ and $R e=500$, this gives $R=1.12$, which is very close to the value given for a single diffusing Lamb-Oseen vortex $R=\sqrt{1+4 \times 30 / R e} \simeq 1.11$. For each different base state, the radius $R$ has been determined by this method.

Using the same pseudo-spectral code, the equations (2.1-2.2) linearized around the basic state have been then integrated for each axial wavenumber $k$ with a white noise as initial conditions and with the same horizontal resolution, i.e. $1024 \times 640$. After a sufficiently long integration time, the perturbation consists solely in the eigenmode with the largest growth rate. Several tests using different resolutions, time steps and box sizes have been carried out in order to check the accuracy of the computations. As seen in the example given in table 1, the growth rate only varies after the fourth significant digit when the box size is doubled, the resolution being fixed (third column) or when the resolution is doubled while keeping the box sizes constant (sixth column). The stability of a vortex pair at time $t=60$ has been also computed for one set of parameters. The growth rate curves obtained for the latter time and $t=30$ differ by less than $2 \%$ demonstrating the independence of the results with respect to the particular time chosen for the base flow.

\subsection{Results}

\subsubsection{Effect of the rotation rate}

Figure $1 \mathrm{~b}$ shows the growth rate $\sigma$ non-dimensionalized by the strain $\Gamma / 2 \pi b^{2}$ of the most unstable mode as a function of the vertical wavenumber $k$ non-dimensionalized by the vortex radius $R$ for different Rossby numbers defined as

$$
R o=\frac{\Gamma}{2 \pi R^{2} \Omega_{b}} .
$$

When $R o=\infty$, there are several independent peaks (dashed dotted lines). The peak at low wavenumber corresponds to the well-known long-wavelength Crow instability (Crow 1970). The dominant wavenumber is $k R \simeq 0.246$, giving a dominant wavelength $\lambda \simeq 7.2 b$ in agreement with the scaling of the Crow's theory (Crow 1970). The maximum growth rate $\sigma \simeq 0.6 \Gamma /\left(2 \pi b^{2}\right.$ ) 
(b)
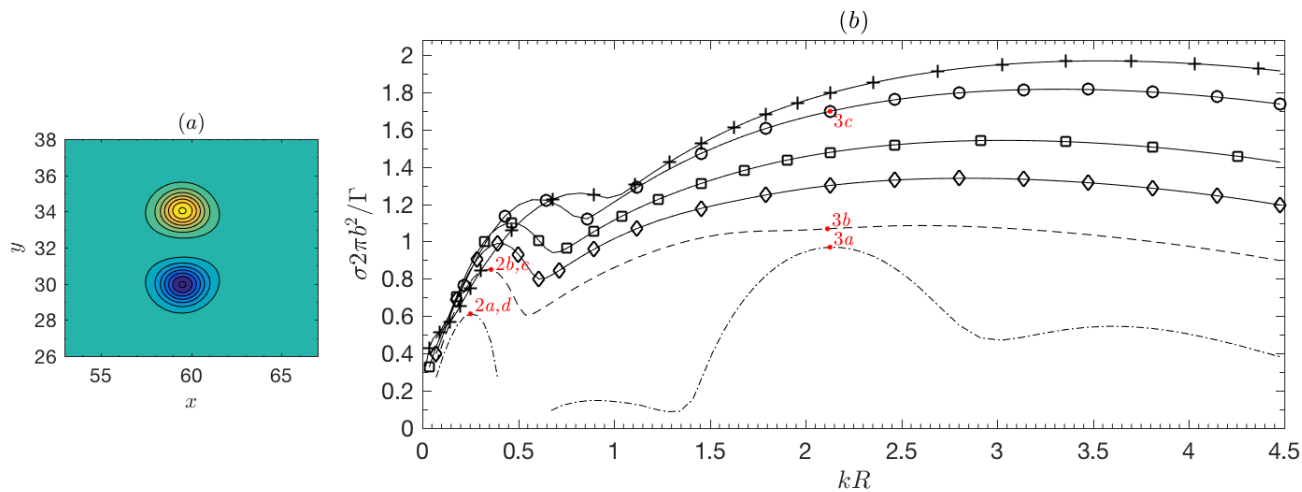

FiguRE 1. (a) Vertical vorticity of the base flow $\omega_{z b}$ for $b=4$ and $R e=500$. The contour level is 0.24 . (b) Non-dimensional growth rate $\sigma 2 \pi b^{2} / \Gamma$ as a function of the axial wavenumber $k R$ for $b=4, R e=500$ and various Rossby numbers: $R o=\infty(-\cdot-), R o=25.55(---), R o=17(-\bigcirc-), R o=12.75(-\square-)$, $R o=8.50(-\circ-), R o=6.38(-+-)$. The labels near the red points indicate the figure number where the corresponding eigenmode is displayed. The symbols are used only to distinguish each curve and do not represent all the data.

is slightly lower than inviscid Crow's theory because $R e$ is moderate. The vertical vorticity $\omega_{z}$ of the dominant eigenmode (figure 2a) displays a dipolar structure in each base vortex. Such perturbation will displace the two vortices as a whole symmetrically as expected for the Crow instability. The second growth rate peak around $k R \simeq 0.9$ (figure $1 \mathrm{~b}$ ) is small and corresponds to an oscillatory instability due to the resonance of azimuthal waves $m=0$ and $m=2$ with the strain (eigenmode not shown) as found for the Lamb-Chaplygin dipole (Billant et al. 1999). The third and fourth peaks for $k R=2.13$ and $k R=3.7$ are related to the first and second antisymmetric modes of the elliptic instability (Kerswell 2002; Leweke et al. 2016) (see the first mode in figure $3 a)$. These wavenumbers agree with the theoretical values predicted for a weakly strained LambOseen vortex in inviscid fluid: $k R=2.26$ and $k R=3.96$ (Sipp \& Jacquin 2003; Leweke et al. 2016). The differences are probably due to viscous effects and finite ellipticity. The viscous effects also explain why the maximum growth rate of the first elliptic peak $\sigma=0.97 \Gamma /\left(2 \pi b^{2}\right)$ is lower than the inviscid theoretical prediction $\sigma=9 / 16 \epsilon=1.41 \Gamma /\left(2 \pi b^{2}\right)$, where $\epsilon=2.52 \Gamma /\left(2 \pi b^{2}\right)$ is the internal strain rate in the case of the Lamb-Oseen vortex (Sipp \& Jacquin 2003; Leweke et al. 2016).

When the background rotation is slightly increased such that $R o=25.55$, the growth rate (dashed line in figure 1b) increases for all wavenumbers and only two distinct peaks are then present. The vertical vorticity of the eigenmode associated to the growth rate peak at low wavenumber is no longer purely symmetric (figure $2 b$ ): the dipolar structure is much weaker on the upper vortex which is cyclonic. This means that the instability will displace almost only the anticyclonic vortex. This asymmetry can be analysed quantatively by decomposing the vertical vorticity $\omega_{z}$ into symmetric and antisymmetric parts as follows

$$
\omega_{z s}=\left(\omega_{z}(x, y)+\omega_{z}\left(x, 2 y_{c}-y\right)\right) / 2, \quad \omega_{z a}=\left(\omega_{z}(x, y)-\omega_{z}\left(x, 2 y_{c}-y\right)\right) / 2,
$$

where $y_{c}$ is the $y$ coordinate of the symmetry plane between the two vortices. The ratio $\Lambda=$ $\int_{0}^{L_{x}} \int_{0}^{L_{y}} \omega_{z s}^{2} \mathrm{~d} x \mathrm{~d} y / \int_{0}^{L_{x}} \int_{0}^{L_{y}} \omega_{z}^{2} \mathrm{~d} x \mathrm{~d} y$ then varies from unity for a purely symmetric mode to zero for a purely antisymmetric mode. As seen in figure $2 \mathrm{~g}, \Lambda$ drops from unity for $R o=\infty$ to $\Lambda=0.73$ for $R o=25.55$.

Similarly, the vertical vorticity of the eigenmode at larger wavenumbers $k R=2.13$ is no longer purely antisymmetric for $R o=25.55$ (figure $3 \mathrm{~b}$ ): this time, the elliptic mode is stronger in the cyclonic vortex than in the anticyclonic vortex whereas a weak band surrounds the core of the 


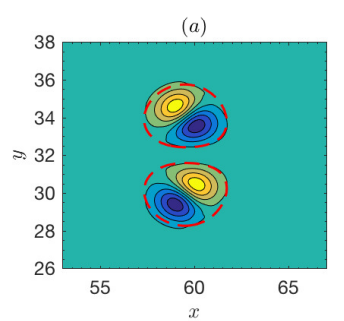

(d)

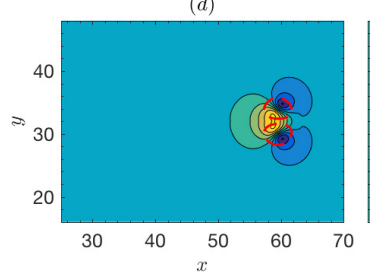

(b)

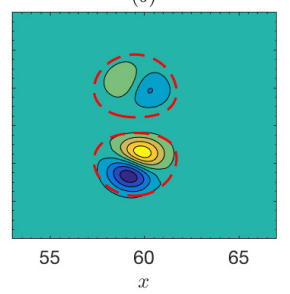

(e)

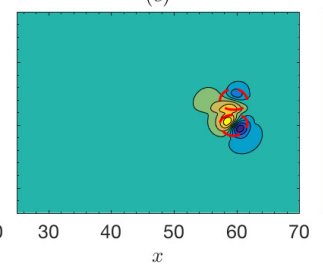

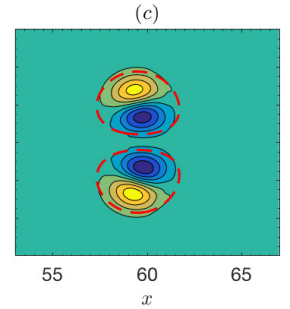

$(f)$

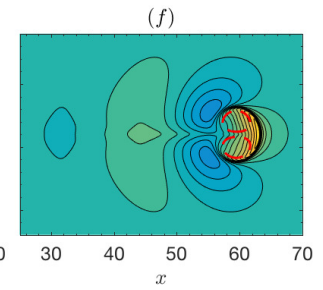

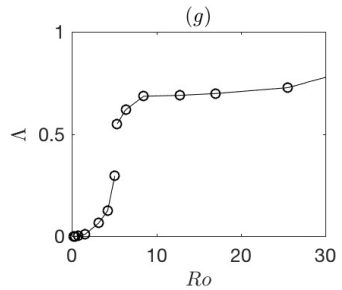

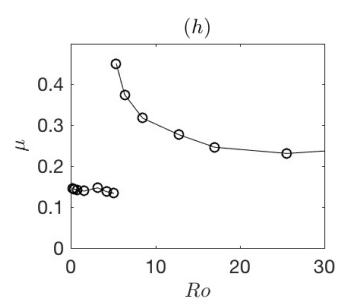

FIGURE 2. Vertical vorticity $(a, b, c)$ and vertical velocity $(d, e, f)$ of the eigenmode corresponding to the first growth rate peak at low wavenumber for $b=4, R e=500$ and different Rossby numbers: $(a, d) R o=\infty$, $(b, e) R o=25.55,(c, f) R o=0.8$. ( $g$ ) Asymmetry ratio $\Lambda$ and $(h)$ ratio $\mu$ of the eigenmode of the first growth rate peak as a function of $R o$. The contour level is 0.24 for $(a, b, c), 0.025$ for $(d, e)$ and 0.0074 for $(f)$. The eigenmode amplitude is normalized so that $\max \left(\left|\omega_{z}\right|\right)=1$. The red dashed lines are the isocontours where the basic state vorticity $\omega_{z b}$ is $0.05 \max \left(\omega_{z b}\right)$.
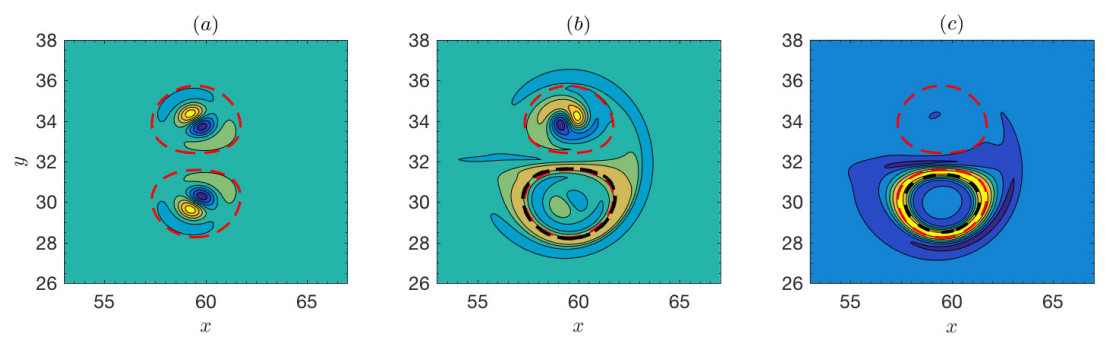

Figure 3. Vertical vorticity of the eigenmode for $k R=2.13$ for $b=4, R e=500$ and different Rossby numbers: (a) $R o=\infty$, (b) $R o=25.55$, (c) $R o=8.50$. The contour level is 0.24 . The red dashed lines are the isocontours where the basic state vorticity $\omega_{z b}$ is $0.05 \max \left(\omega_{z b}\right)$. The black thick dashed line in $(b, c)$ represents the contour where $\omega_{z b}+2 \Omega_{b}$ changes sign.

latter vortex. When the Rossby number is decreased from $R o=25.55$ to $R o=8.5$, this mode on the anticyclonic vortex becomes strong while the elliptic mode on the cyclonic vortex fades out (figure 3c). Concomitantly, the growth rate continues to globally increase monotonically in the band $k R=[1,4]$ (figure $1 \mathrm{~b}$ ) until $R o \simeq 4.25$ and then it decreases and goes to zero around $R o=1.35 \pm 0.05$ (not shown). As seen in figure $3 \mathrm{~b}, \mathrm{c}$, the mode on the anticyclonic vortex arises in the vicinity of the line where the absolute vorticity $\omega_{z b}+2 \Omega_{b}$ changes sign (thick black dashed line). As shown by Sipp \& Jacquin (2000) and Gallaire \& Rousset (2006), this is an inviscid criterion for the centrifugal instability in non-axisymmetric rotating fluids. The critical value $R o \simeq 1.35$ is also in good agreement with the critical Rossby number $R o=1$ predicted by this criterion for the stabilization of the centrifugal instability. Viscous effects are certainly responsible for the difference.

The growth rate curves for smaller Rossby numbers down to $R o=0.2$ are displayed on figure 4a. Only a focus on small wavenumbers is shown. The growth rate for $R o=6.38$ is plotted again to serve as reference (solid line with + symbols). While the growth rate for any wavenumber was 

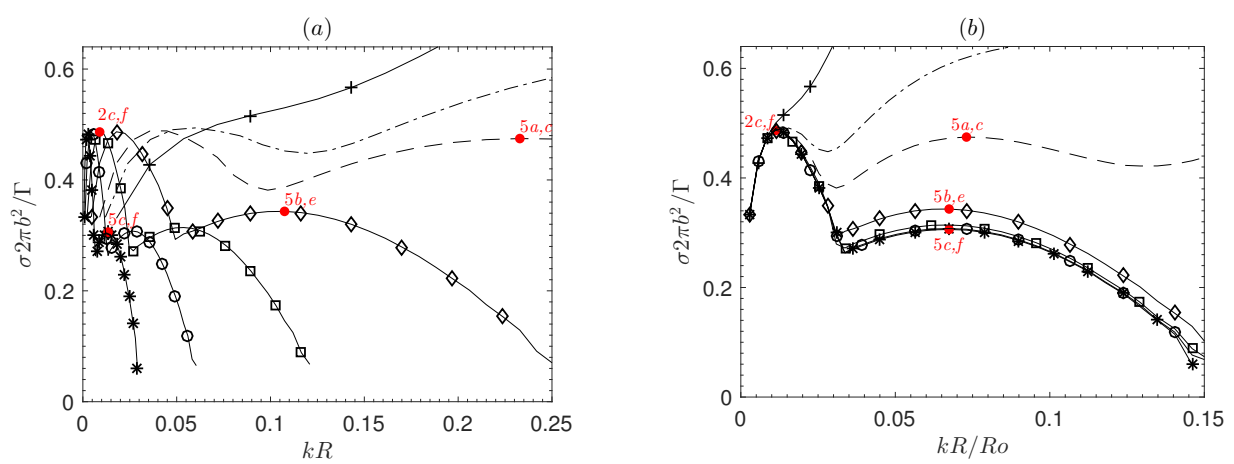

FiguRe 4. Non-dimensional growth rate $\sigma 2 \pi b^{2} / \Gamma$ as a function of the axial wavenumber $k R(a)$ and $k R / R o$ (b) for $b=4, R e=500$ and various Rossby numbers: $R o=6.38(-+-), R o=4.25(-\cdot-), R o=3.19$

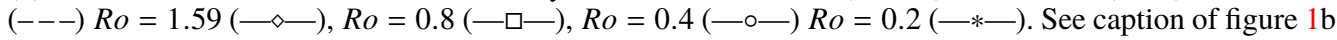
for other details.

globally increasing as $R o$ decreases when $R o \geqslant 8.5$ (figure $1 \mathrm{~b}$ ), a different behaviour is observed in figure $4 \mathrm{a}$ when $R o \leqslant 4.25$. Indeed, the growth rate peak located around $k R \simeq 0.8$ for $R o=6.38$ disappears but another peak emerges at lower wavenumber $k R \simeq 0.06$ for $R o=4.25$. As $R o$ is further decreased, the maximum growth rate of this peak remains constant. In contrast, the corresponding most amplified wavenumber decreases dramatically as $R o$ decreases. Rescaling $k R$ by $R o$ (figure $4 \mathrm{~b}$ ), we can see that all the growth rate curves collapse for low wavenumbers near the first peak. When $R o \leqslant 0.8$, the collapse is almost perfect for the whole growth rate curves. This proves that the vortex remains unstable to three-dimensional perturbations as $R o \rightarrow 0$ but the dominant wavenumber scales like $R o$. The maximum growth rate is $\sigma \simeq 0.5 \Gamma /\left(2 \pi b^{2}\right)$, i.e. of the same order as the maximum growth rate of the Crow instability in non-rotating fluid.

The associated eigenmode is almost antisymmetric (figures 2c) and consists in a dipolar structure in each base vortex. Hence, this instability will displace the two vortices as a whole in antisymmetric directions. As displayed in figure $2 \mathrm{~g}$, the asymmetry ratio behaves like $\Lambda \simeq$ $0.006 R o^{2}$ for $R o \lesssim 1$ so that the eigenmode tends to a purely antisymmetric mode when $R o=0$. For $R o \leqslant 3.19$, a second subdominant peak appears at slightly larger wavenumbers (figure 4a). The corresponding eigenmode resembles the one of the first peak except that it is quasisymmetric (figure 5) like for the Crow instability. The asymmetry ratio tends to unity as $R o \rightarrow 0$ like $\Lambda \simeq 1-0.008 R o^{2}$ (figure $5 \mathrm{~g}$ ). It is also interesting to look at the vertical velocity field of these eigenmodes (figures $2 \mathrm{f}$ and $5 \mathrm{~d}$,e,f). Compared to $R o=\infty$ (figure $2 \mathrm{~d}$ ), $u_{z}$ extends well outside the vortex cores and exhibits a wave train in the wake of the vortex pair (note the different scales in figures $2 \mathrm{~d}, \mathrm{e}, \mathrm{f}$ and $5 \mathrm{~d}, \mathrm{e}, \mathrm{f}$ compared to figures $2 \mathrm{a}, \mathrm{b}, \mathrm{c}$ and $5 \mathrm{a}, \mathrm{b}, \mathrm{c})$. In addition, it is worth to point out that the ratio between the maximum vertical and horizontal velocities

$$
\mu=\frac{\max \left(\left|u_{z}\right|\right)}{\max \left(\sqrt{\left(u_{x}^{2}+u_{y}^{2}\right) / 2}\right)},
$$

remains of order unity as Ro goes to zero (figures $2 \mathrm{~h}$ and $5 \mathrm{~h}$ ).

Even if we have focussed on the region of small vertical wavenumbers in figure 4, we stress that we have not observed any other type of instability at higher wavenumbers when $R o \leqslant 1$.

\subsubsection{Effects of the separation distance and Reynolds number}

Figure $6 \mathrm{a}$ shows the growth rate non-dimensionalized by $\Gamma / 2 \pi R^{2}$ for different separation distances $b$ for a fixed low Rossby number $R o=0.4$ which belongs to the self-similar regime found above. The maximum growth rate of the two peaks and dominant wavenumbers decrease as $b$ increases, meaning that the instabilities weaken as the vortices are more distant. The inset 


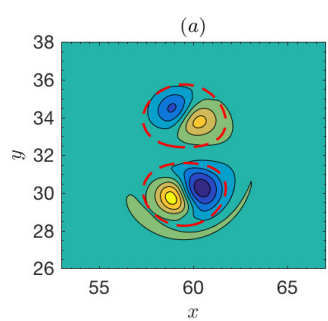

(d)

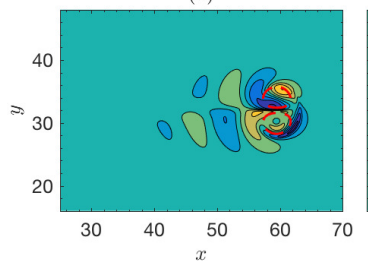

(b)

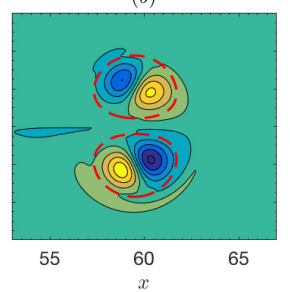

(e)

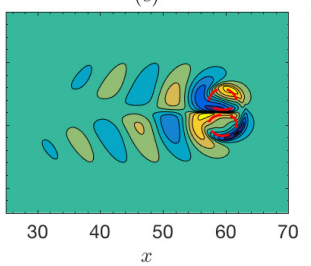

(c)

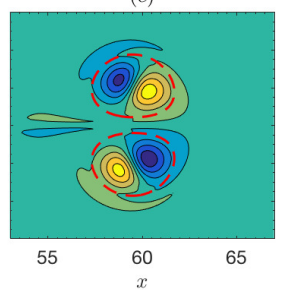

(f)

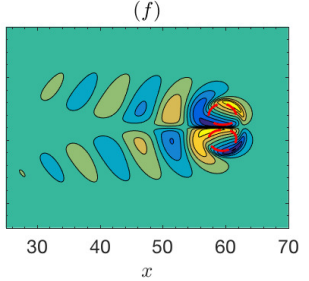

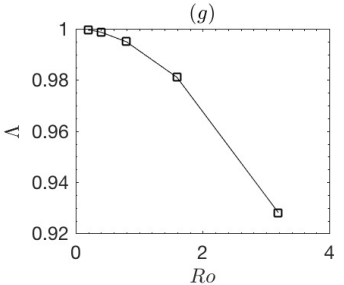

(h)

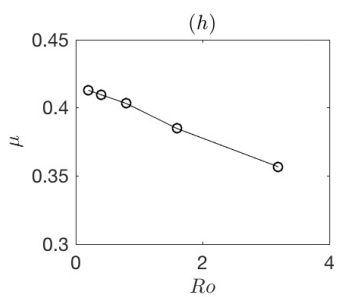

FigURE 5. Vertical vorticity $(a, b, c)$ and vertical velocity $(d, e, f)$ of the eigenmode corresponding to the second growth rate peak for $b=4, R e=500$ and different Rossby numbers: $(a, d) R o=3.19,(b, e) R o=1.59$, $(c, f) R o=0.2$. The contour level is 0.24 for $(a, b, c)$ and 0.038 for $(d, e, f)$. $(g)$ Asymmetry ratio $\Lambda$ and $(h)$ ratio $\mu$ of the eigenmode of the second peak as a function of $R o$. In $(a, b, c, d, e, f)$, the red dashed lines are the isocontours where the basic state vorticity $\omega_{z b}$ is $0.05 \max \left(\omega_{z b}\right)$.
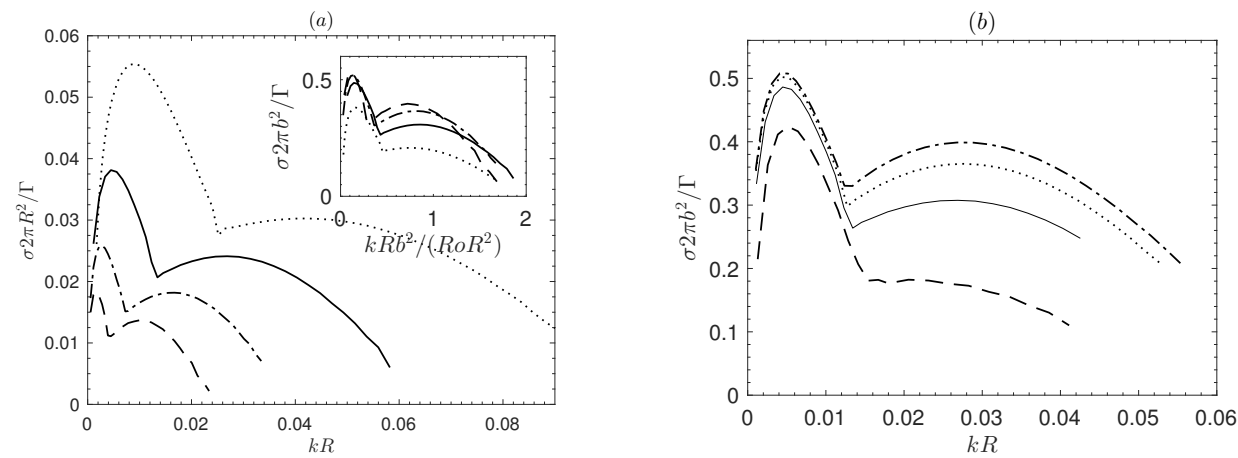

FiguRe 6. (a) Non-dimensional growth rate $\sigma 2 \pi R^{2} / \Gamma$ as a function of the axial wavenumber $k R$ for $R e=500, R o=0.4$ and different separation distance $b=3(\cdots), b=4(-), b=5(-\cdot-), b=6(---)$. The inset displays the same curves in terms of the non-dimensional growth rate $\sigma 2 \pi b^{2} / \Gamma$ and wavenumber $k R b^{2} /\left(R o R^{2}\right)$. (b) Non-dimensional growth rate $\sigma 2 \pi b^{2} / \Gamma$ as a function of $k R$ for $b=4$ and $R e=200$, $R o=0.30(---), R e=500, R o=0.4(-), R e=1000, R o=0.44(\cdots), R e=2000, R o=0.47(-\cdot-)$. In all the cases, the rotation rate is fixed to $\Omega_{b}=2$ but the Rossby number varies slightly since the radius $R$ increases as $R e$ decreases.

in figure 6a shows the same curves but when the growth rate is rescaled by the strain and the wavenumber $k$ by $b^{2} /(R o R)$. We can see that the growth rate peaks are approximately aligned indicating that, together with the dependence with the Rossby number found above, the dominant wavenumber scales like $R k_{\text {max }} \simeq 0.15 R o_{b}$ where $R o_{b}=\Gamma /\left(2 \pi b^{2} \Omega_{b}\right)$ is a Rossby number based on the separation distance $b$ instead of the vortex radius $R$. The corresponding wavelength scaling $\lambda_{\text {max }} \simeq 42 R / R o_{b}$ differs from the Crow instability one's $\lambda_{\max } \propto b$. Furthermore, the maximum growth rate of the Crow instability scales like the strain $\Gamma /\left(2 \pi b^{2}\right)$ while the inset of figure 6 a shows that this scaling does not collapse well the curves. A better collapse, but still rather approximate, could be obtained by plotting $\sigma b^{n}$ with an exponent $n$ around 3/2 (not shown). 
Similarly, the dominant wavelength associated to the subdominant growth rate peak scales like $\lambda_{\text {max }} \simeq 7 R / R o_{b}$.

Finally, the effect of the Reynolds number is investigated in figure $6 \mathrm{~b}$ for $b=4$ and a fixed rotation rate $\Omega_{b}=2$ for which $R o=0.4 \pm 0.1$. The growth rate increases with $R e$ and tends to saturate to finite values as $R e$ becomes large, indicating that the instability is inviscid.

\section{Direct Numerical simulations}

To complement the linear stability analyses, we have performed some Direct Numerical Simulations of the evolution of the quasi-antisymmetric displacement instability that is dominant for low Rossby number. The aims are to confirm that the instability does indeed lead to a threedimensionalization of the vortex pair and to determine whether its nonlinear evolution continues to follow the same dependence on the Rossby number as that found in $\S 2$. However, a detailed analysis of the nonlinear saturation mechanism is left for future studies.

These DNS have been carried out for two Rossby numbers $R o=1.53$ and $R o=0.76$ for the Reynolds number $R e=500$. The separation distance has been taken as $b=3$ because it leads to a smaller most amplified wavelength than for $b=4$ (see §2.2.2) and thus requires a shorter computational domain in the vertical direction. For the sake of the computational cost, the horizontal sizes of the domain $L_{x} \times L_{y}$ have been reduced to $60 \times 31.5$ keeping the same horizontal mesh sizes. Table 1 (first column) shows indeed that the growth rate varies by less than $1 \%$ compared to the reference value obtained with the wider domain (second column).

The initial velocity field corresponds to the sum of the velocities of the base state and a perturbation consisting in the dominant eigenmode. The amplitude of the perturbation has been fixed such that its maximum vertical vorticity is $5 \%$ of the maximum vertical vorticity of the base flow. The vertical size of the domain $L_{z}$ has been adjusted to the most amplified wavelength $\lambda_{\max }$ for each Rossby number, i.e. $L_{z}=196.43$ for $R o=1.53$ and $L_{z}=392.85$ for $R o=0.76$. The mesh sizes have been set to be almost isotropic. Thereby, the resolutions are $N_{x} \times N_{y} \times N_{z}=$ $512 \times 320 \times 1600$ for $R o=1.53$ and $512 \times 320 \times 3200$ for $R o=0.76$. The truncation has been reduced to $9 / 10$ of the modes along each direction. The convergence has been checked by increasing the resolution to $768 \times 480 \times 2400$ for $R o=1.53$. The velocity at each point common to both resolutions have been found to differ by less than $1 \%$ at any time.

Figure 7 shows three-dimensional contours and horizontal cross-sections of the vertical vorticity for $R o=0.76$ at different times. A movie is available in the supplementary material. While the vortex pair looks straight at $t=0$ (figure $7 \mathrm{a}$ ) since the perturbation is initially small, a vertical bending of the vortices becomes clearly visible at $t=100$ (figure 7e). In the horizontal cross-sections at $z=L_{z} / 2$ (figure $7 \mathrm{~h}$ ) and $z=L_{z}$ (figure $7 \mathrm{f}$ ), we see that the vortex pair propagates obliquely and symmetrically with respect to the $x$ direction. In contrast, the vortex pair continues to propagate in the $x$ direction at $z=3 L_{z} / 4$ (figure $7 \mathrm{~g}$ ). Such behaviour, which is consistent with the structure of the vertical vorticity of the eigenmode (figure $2 \mathrm{c}$ ), is similar to the zigzag instability one's in strongly stratified fluid (Billant \& Chomaz 2000; Deloncle et al. 2008). Note that the simulation is performed in the traveling reference frame where the vortex pair is steady at $t=0$. Since the vortex pair slows down due to viscous effects, it thus moves backward in the $x$ direction in this reference frame.

At $t=130$ (figure 7i), the vertical deformation has amplified and the vortex pair is now traveling in almost opposite $y$ direction at $z=L_{z} / 2$ (figure 7l) and $z=L_{z}$ (figure $7 \mathbf{j}$ ). In between, at $z=3 L_{z} / 4$ (figure $7 \mathrm{k}$ ), the vortex pair has kept its initial direction of propagation but two tongues of opposite vertical vorticity wrap the external part of each vortex. Such emergence of vertical vorticity is due to the stretching of the background vorticity by the vertical velocity, i.e. the term $2 \Omega_{b} \partial_{z} u_{z}$. The interactions between these tongues and the vortex cores initiate a breakdown of the vortex pair into smaller three-dimensional structures. Indeed, we see in figure 

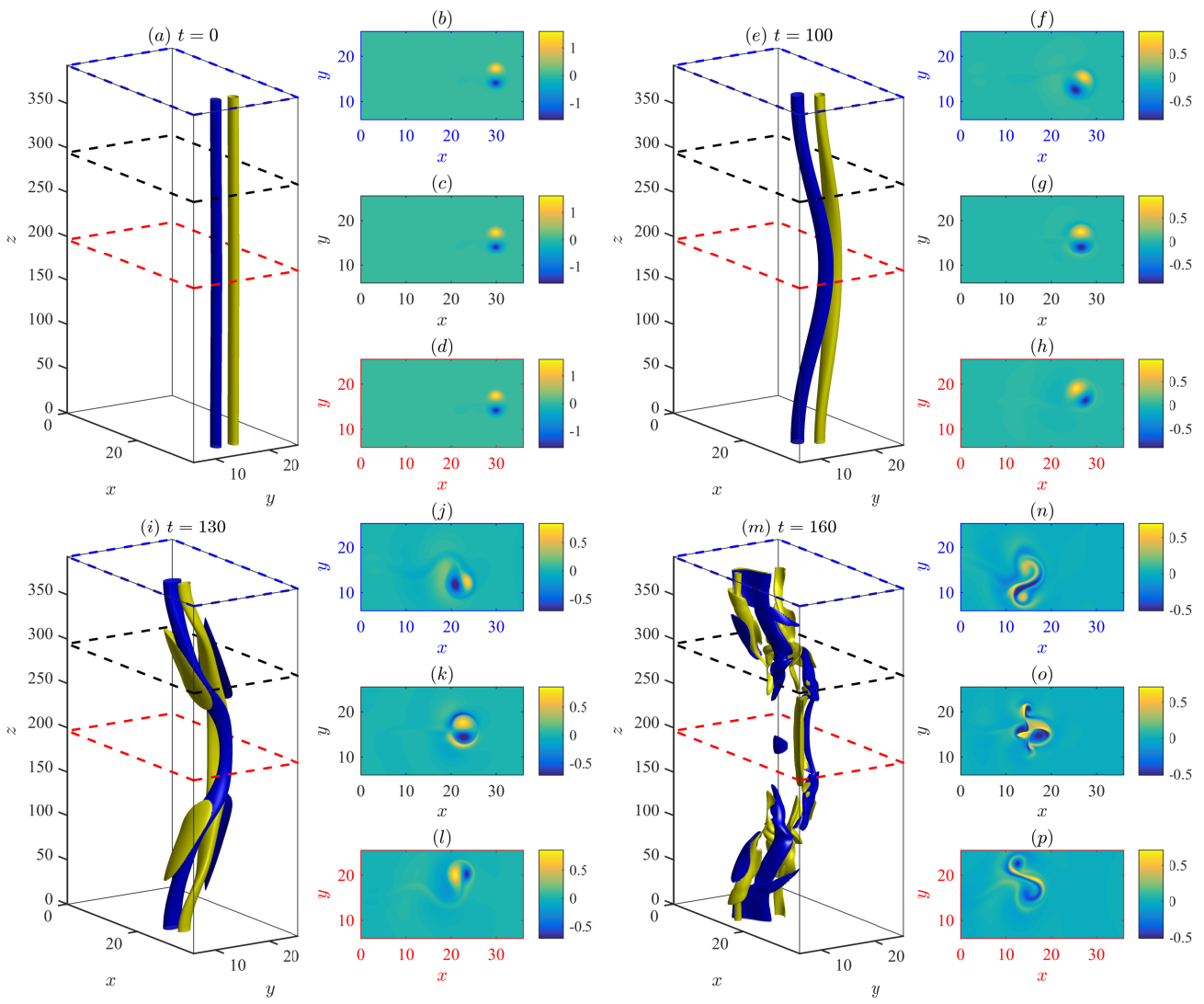

Figure 7. $(a, e, i, m)$ Three-dimensional contours of the vertical vorticity $\omega_{z}$ at different times in a Direct Numerical Simulation for $R o=0.76, b=3$ and $R e=500$. Yellow and blue contours represent respectively plus and minus $50 \%$ of the vertical average of the maximum vertical vorticity in each horizontal plane. For each time, three horizontal cross-sections of the vertical vorticity in the planes $z=L_{z} / 2(d, h, l, p)$, $z=3 L_{z} / 4(c, g, k, o)$ and $z=L_{z}(b, f, j, n)$ are displayed. The location of these cross-sections are also indicated by dashed lines with different color in $(a, e, i, m)$. The times shown are: $(a, b, c, d) t=0,(e, f, g, h)$ $t=100,(i, j, k, l) t=130,(m, n, o, p) t=160$. Note that only a portion of the computational domain is shown.

$7 \mathrm{~m}$, at $t=160$, that the vortex pair becomes fully three-dimensional and the initial vortex cores of the pair are strongly disorganized in the horizontal cross-sections (figure 7n,o,p).

Figure 8a displays the evolutions of the total horizontal and vertical kinetic energies $E_{k h}=$ $\left\langle u_{x}^{2}+u_{y}^{2}\right\rangle / 2$ (divided by 20 for convenience) and $E_{k v}=\left\langle u_{z}^{2}\right\rangle / 2$, where $\langle\cdot\rangle=\left(1 / L_{z}\right) \int_{V} \mathrm{dV}$ is the volume integration per unit wavelength. In this way, these quantities are independent of the sizes of the computational box. The vertical kinetic energy (black dashed line with circle symbols) first grows exponentially and then saturates for $t>150$ to a value of order unity. In contrast, the horizontal kinetic energy (black solid line with circle symbols) always slowly decays. Although the total horizontal kinetic energy is much larger than the vertical one, the inset in figure 8a shows that the ratio $\mu$ (see 2.5 ) is of order unity at saturation, i.e. the maximum vertical and horizontal velocities are of the same order of magnitude.

The corresponding evolutions of the total vertical and horizontal enstrophies $Z_{v}=\left\langle\omega_{z}^{2}\right\rangle / 2$ and 

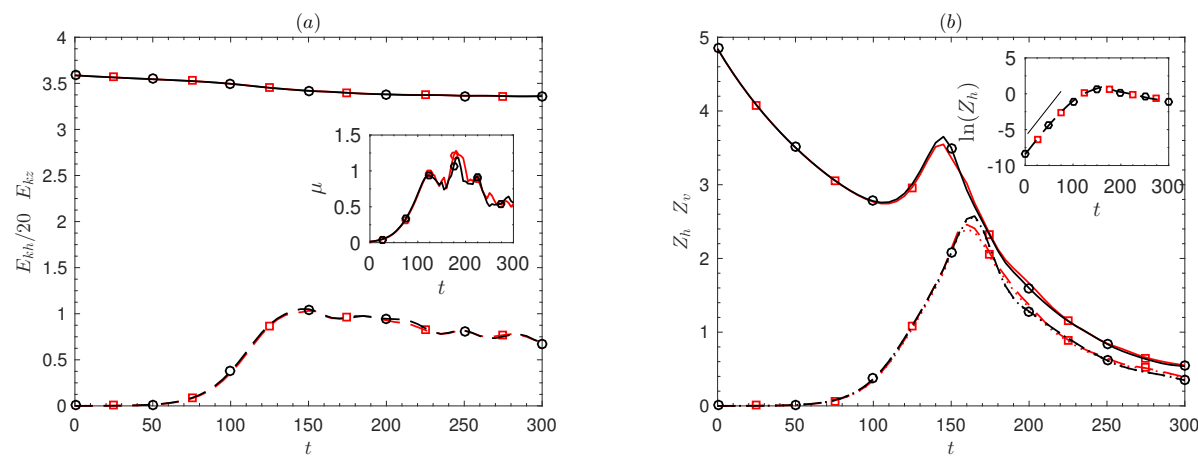

Figure 8. Evolution of the $(a)$ kinetic energy and $(b)$ enstrophy for two Rossby numbers: $R o=0.76$ (black lines with circle symbols) and $R o=1.53$ (red lines with square symbols) for $b=3$ and $R e=500$. In $(a)$, the different lines correspond to horizontal and vertical kinetic energies $E_{k h}(-)$ and $E_{k v}(---)$. Note that $E_{k h}$ has been divided by a factor 20 . In $(b)$, the lines represent vertical enstrophy $Z_{v}(-)$, horizontal enstrophy $Z_{h}(---)$ and $\left\langle\left(\partial_{y} u_{z}\right)^{2}+\left(\partial_{x} u_{z}\right)^{2}\right\rangle / 2(\cdots)$. The inset in $(a)$ represents the evolution of the ratio $\mu$ defined in (2.5). The inset in (b) displays the evolution of the logarithm of the horizontal enstrophy $Z_{h}$. The straight solid line represents an exponential growth at rate $2 \sigma$.

$Z_{h}=\left\langle\omega_{x}^{2}+\omega_{y}^{2}\right\rangle / 2$ are shown in figure $8 \mathrm{~b}$. The horizontal enstrophy $Z_{h}$ (black dashed line with circle symbols) grows exponentially until $t=160$. The inset in figure $8 \mathrm{~b}$ shows that the growth rate of $Z_{h}$ is in good agreement with the linear stability analysis. The horizontal enstrophy saturates when reaching a value comparable to the vertical enstrophy $Z_{v}$ (black solid line with circle symbols) and then decays. In fact, the horizontal vorticity corresponds mostly to the horizontal shear of the vertical velocity, i.e. $\omega_{x} \simeq \partial_{y} u_{z}, \omega_{y} \simeq-\partial_{x} u_{z}$. Indeed, the black dotted line, which displays $\left\langle\left(\partial_{y} u_{z}\right)^{2}+\left(\partial_{x} u_{z}\right)^{2}\right\rangle / 2$, is almost superposed to the curve of $Z_{h}$ (figure $8 \mathrm{~b}$ ). The vertical enstrophy, while decaying by viscous diffusion for $t<100$, re-increases momentarily between $t \simeq 110$ and $t \simeq 150$. This is related to the appearance of the opposite vertical vorticity tongues (figure $7 \mathrm{i}$ ).

The three-dimensional evolution of the vertical vorticity for the Rossby number $R o=1.53$ is almost identical to the one for $R o=0.76$ (see the movie in the supplementary material). The evolutions of kinetic energies and enstrophies for $R o=1.53$ are also plotted in figure 8 (red lines with square symbols). Remarkably, they are almost superposed to those for $R o=0.76$. This demonstrates that the self-similarity evidenced in the linear stability analysis applies also to the non-linear evolution of the instability. Altogether, this three-dimensional instability will therefore develop similarly whatever $R o \lesssim 1$ in unbounded flows.

\section{Scaling analysis}

In section 2, we have found that the dominant wavelength of the three-dimensional instability of a counter-rotating vortex pair is inversely proportional to the Rossby number when $R o \lesssim 1$. Here, we generalize this result by showing that this scaling derives from a self-similarity of (2.1-2.2) when the Rossby number is small. To this end, we consider a flow with characteristic horizontal and vertical velocity scales $U$ and $W$ and typical horizontal and vertical lengthscales $L_{h}$ and $L_{v}$. The Rossby number $R o=U /\left(2 \Omega_{b} L_{h}\right)$ is assumed to be small and the fluid is considered to be unbounded. For the sake of clarity and simplicity, we consider an inviscid fluid but it will be shown at the end that the following analysis applies equally to viscous flows.

In order to scale the equations (2.1-2.2) with $v=0$, we define dimensionless quantities denoted 
with prime as follows

$$
\boldsymbol{u}_{h}=U \boldsymbol{u}_{h}^{\prime}, \quad u_{z}=W u_{z}^{\prime}, \quad p=2 \rho_{0} \Omega_{b} U p^{\prime}, \quad x=L_{h} x^{\prime}, \quad y=L_{h} y^{\prime}, \quad z=L_{v} z^{\prime}, \quad t=\frac{L_{h}}{U} t^{\prime} .
$$

where the subscript $h$ is used to denote the horizontal components of any vector. The magnitude of the pressure has been fixed so as to balance the Coriolis force. The characteristic time scale has been chosen to be the advective timescale along the horizontal $L_{h} / U$. Such hypothesis excludes fast inertial waves with typical frequency $O\left(\Omega_{b}\right)$ but not those with a slow frequency of the order of $L_{h} / U$. The primes will be dropped in the following in order to keep the notation simple.

The resulting dimensionless equations are

$$
\begin{aligned}
R o\left[\partial_{t} \boldsymbol{u}_{h}+\boldsymbol{u}_{h} \cdot \boldsymbol{\nabla}_{h} \boldsymbol{u}_{h}+\alpha \delta u_{z} \partial_{z} \boldsymbol{u}_{h}\right]+\boldsymbol{e}_{z} \times \boldsymbol{u}_{h} & =-\boldsymbol{\nabla}_{h} p, \\
R o\left[\partial_{t} u_{z}+\boldsymbol{u}_{h} \cdot \boldsymbol{\nabla}_{h} u_{z}+\alpha \delta u_{z} \partial_{z} u_{z}\right] & =-\frac{\delta}{\alpha} \partial_{z} p, \\
\boldsymbol{\nabla}_{h} \cdot \boldsymbol{u}_{h}+\alpha \delta \partial_{z} u_{z} & =0,
\end{aligned}
$$

where $\alpha=W / U$ and $\delta=L_{h} / L_{v}$. It is also convenient to write the equation for the dimensionless vertical vorticity $\omega_{z}$

$$
R o\left[\partial_{t} \omega_{z}+\boldsymbol{u}_{h} \cdot \nabla_{h} \omega_{z}+\alpha \delta u_{z} \partial_{z} \omega_{z}\right]=\alpha \delta\left[R o\left(\partial_{z} u_{x} \partial_{y} u_{z}-\partial_{z} u_{y} \partial_{x} u_{z}+\omega_{z} \partial_{z} u_{z}\right)+\partial_{z} u_{z}\right]
$$

In the following, we further assume a priori that the vertical Rossby number is small

$$
R o_{v}=\frac{W}{2 \Omega_{b} L_{v}} \equiv \operatorname{Ro} \alpha \delta \ll 1,
$$

but no other assumption on the individual orders of magnitude of $\alpha$ and $\delta$ will be used. This assumption together with $R o \ll 1$ are sufficient to deduce the geostrophic balance from (4.2)

$$
\boldsymbol{u}_{h}=-\boldsymbol{\nabla} \times p \boldsymbol{e}_{z}+O\left(R o, R o_{v}\right) .
$$

The vertical vorticity is therefore

$$
\omega_{z}=\Delta_{h} p+O\left(R o, R o_{v}\right) .
$$

and the divergence equation (4.4) becomes

$$
\alpha \delta \partial_{z} u_{z}+O\left(R o, R o_{v}\right)=0 .
$$

Hence, (4.9) implies the order of magnitude relation:

$$
\alpha \delta \leqslant O(R o),
$$

confirming the hypothesis (4.6) since $R o_{v}=R o \alpha \delta \leqslant R o^{2}$. By inserting (4.8) into the equation for the vertical vorticity (4.5) and by taking into account (4.10), we obtain at leading orders

$$
R o\left[\partial_{t} \Delta_{h} p+\boldsymbol{u}_{h} \cdot \nabla_{h} \Delta_{h} p\right]=\alpha \delta \partial_{z} u_{z}+O\left(R o^{2}\right) .
$$

By using (4.10), the momentum equation along the vertical (4.3) can be also simplified to

$$
R o\left(\partial_{t} u_{z}+\boldsymbol{u}_{h} \cdot \nabla_{h} u_{z}\right)=-\frac{\delta}{\alpha} \partial_{z} p+O\left(R o^{2}\right) .
$$

Remarkably, this equation combined with (4.11) close the problem and allow to find the leading order solution in terms of $\left(p, u_{z}\right)$. Hence, the system has been reduced from 4 equations and variables to only 2 by simply using the two relations imposed by the geostrophic balance (4.7). It should also be pointed out that no a priori assumption on the magnitudes of $\alpha$ and $\delta$ has been necessary to derive these equations except (4.6). 
As shown by Julien \& Knobloch (2007) and Nazarenko \& Schekochihin (2011), the application of the dominant balance or critical balance principles, respectively, to (4.11-4.12) implies $\alpha \delta=$ $O(R o)$ and $\delta / \alpha=O(R o)$. Note that the critical balance principle imposes that the typical time scale of waves and advective motions should be of the same order (Nazarenko \& Schekochihin 2011). These principles both lead to $\alpha=O(1)$ and $\delta=O(R o)$. However, the key point developed herein is that it is not necessary to invoke the dominant balance or critical balance principles to obtain these relations. They can be derived rigorously from an invariance of the equations (4.11) and (4.12). Indeed, if we do not use these principles and still consider that $\delta$ and $\alpha$ are arbitrary and unknown, we can eliminate the Rossby number $R o, \delta$ and $\alpha$ from (4.11) and (4.12) by the simple rescaling $\tilde{z}=z R o / \delta$ and $\tilde{u}_{z}=\alpha u_{z}$

$$
\begin{gathered}
\partial_{t} \Delta_{h} p+\boldsymbol{u}_{h} \cdot \nabla_{h} \Delta_{h} p=\partial_{\tilde{z}} \tilde{u}_{z}, \\
\partial_{t} \tilde{u}_{z}+\boldsymbol{u}_{h} \cdot \nabla_{h} \tilde{u}_{z}=-\partial_{\tilde{z}} p,
\end{gathered}
$$

so that all the non-dimensional parameters have disappeared. This rescaling gives the same scaling law for the vertical scale $L_{v}$ as the critical or dominant balances: $\delta=R o$, i.e.

$$
L_{v} \sim L_{h} / R o .
$$

Similarly, the rescaling of the vertical velocity is equivalent to state that the orders of magnitude of the vertical and horizontal velocities are equal: $\alpha=1$, i.e. $W=U$. Therefore, the vertical lengthscale in rapidly rotating flows is set by a self-similarity of the equations for $R o \ll 1$ like in strongly stratified fluids (Billant \& Chomaz 2001).

A posteriori, it is straightforward to remark that the above self-similarity continues to hold in presence of viscous effects because the laplacian of the viscous operator $\Delta$ can be approximated by $\Delta_{h}$ since $\delta^{2} \ll 1$. This explains why the scaling law (4.15) has been found to be well verified in $\S 2$ even for a moderate Reynolds number $R e=500$.

The reduced equations (4.11-4.12), or equivalently (4.13-4.14), with the addition of viscous effects are identical to those obtained for rapidly rotating convection based on the dominant balance principle (Julien et al. 1998; Julien \& Knobloch 2007) or for rotating turbulence by invoking the critical balance principle (Nazarenko \& Schekochihin 2011). Nevertheless, we believe that the present derivation based on a self-similarity is less heuristic and more general, thereby extending their range of applicability.

Besides the scaling law (4.15) for the vertical lengthscale, we emphasize that two other observations made in $\$ 2$ are fully consistent with this scaling analysis: first, the growth rate (figure $4 \mathrm{~b}$ ) and time evolution (figure 8) are independent of the Rossby number for low Rossby number in agreement with the assumption that the characteristic timescale is $L_{h} / U$. Second, the ratio (2.5) is of order unity (figures $2 \mathrm{~h}, 5 \mathrm{~h}$ and $8 \mathrm{a}$ ) consistently with the scaling $W=U$. Furthermore, these scaling laws imply $\omega_{x}=\partial_{y} u_{z}+O(R o), \omega_{y}=-\partial_{x} u_{z}+O(R o)$ as observed in $\S 3$.

\section{Conclusions and discussion}

We have investigated the stability of a pair of counter-rotating vertical vortices in a fluid rotating about the vertical axis with a particular focus on the limit of large rotation. The main question being: is this flow totally stable to three-dimensional perturbations for large rotation rate?, as generally argued based on the Taylor-Proudman theorem.

When the background rotation is slightly increased from zero, the Crow and elliptic instabilities quickly loose their symmetry with respect to the plane between the two vortices. For moderate rotation rate, the centrifugal instability arises on the anticyclonic vortex. For large rotation rate, we have found a new instability of inviscid nature that consists in quasi-antisymmetric displacements 
of the two vortices. Its growth rate is independent of the Rossby number when $R o \lesssim 1$ while its dominant wavelength scales like $\lambda \simeq 42 R / R o_{b}$, where $R o_{b}=R o R^{2} / b^{2}$. A subdominant quasisymmetric displacement instability is also present at smaller wavelengths. A similar scaling in terms of $R o$ has been reported for the width of the unstable vertical wavenumber bands of the instability of a rotating horizontal shear layer (Arobone \& Sarkar 2012) and of an inertial wave triadic instability (van Kan \& Alexakis 2020). However, the dominant instability remains always two-dimensional in these two cases.

Direct Numerical Simulations of the non-linear evolution of the dominant instability for low Rossby number reveals that it triggers a full three-dimensional breakdown of the vortex pair. Furthermore, the complete evolution of the instability follows the self-similarity with respect to the Rossby number, i.e. not only its linear development.

In brief, we have found that some three-dimensional instabilities continue to exist with a finite growth rate for vanishing Rossby number but their wavelength tends to infinity. Such threedimensional behaviour is consistent with the observations of a dimensional transition of rapidly rotating turbulence when the vertical scale is large compared to the horizontal scale (Deusebio et al. 2014; van Kan \& Alexakis 2020). These results imply that the Taylor-Proudman theorem can be strictly valid only for vertically bounded flows. In contrast, there is no contradiction with the proof of Gallet (2015) that two-dimensional turbulent flows are stable to three-dimensional perturbations for large rotation since a vertically bounded flow is assumed.

In the light of the stability analysis, we have performed a general scaling analysis for rapidly rotating flows. Using only the hypotheses that the Rossby number is small and the flow unbounded, we have been able to derive the scaling law for the vertical lengthscale $L_{v} \propto L_{h} / R o$ directly from a self-similarity of the Navier-Stokes equations. This property generalizes and strengthens the previous heuristic multiscales analyses of Julien et al. (1998), Julien \& Knobloch (2007) and Nazarenko \& Schekochihin (2011).

Interestingly, the growth rate of the displacement instabilities evidenced here does not scale precisely with the external strain $\Gamma /\left(2 \pi b^{2}\right)$ unlike the Crow instability. The understanding of this feature is left for future work. It would be also of interest to study more precisely their nonlinear evolutions and to analyse the stability of other vortex configurations since there is no reason to believe that these instabilities are specific to a counter-rotating vortex pair.

I gratefully thank D. Guy and V. Toai for technical assistance, the anonymous referees for their comments and S. Galtier and P. Augier for helpful discussions on the scaling analysis. This work was performed using HPC resources from GENCI-IDRIS (Grant 2020-A0082A07419).

The author reports no conflict of interest.

\section{REFERENCES}

Alexakis, A \& Biferale, L 2018 Cascades and transitions in turbulent flows. Physics Reports 767, 1-101. Arobone, E. \& SARkar, S. 2012 Evolution of a stratified rotating shear layer with horizontal shear. part i. linear stability. J. Fluid Mech. 703, 29-48.

Billant, P., Brancher, P. \& Chomaz, J.-M. 1999 Three-dimensional stability of a vortex pair. Phys. Fluids 11, 2069-2077.

Billant, P. \& Chomaz, J.-M. 2000 Experimental evidence for a new instability of a vertical columnar vortex pair in a strongly stratified fluid. J. Fluid Mech. 418, 167-188.

Billant, P. \& Сhомaz, J.-M. 2001 Self-similarity of strongly stratified inviscid flows. Phys. Fluids 13, $1645-1651$.

Cambon, C, Rubinstein, R \& Godeferd, FS 2004 Advances in wave turbulence: rapidly rotating flows. New Journal of Physics 6 (1), 73.

Crow, S. C. 1970 Stability theory for a pair of trailing vortices. A.I.A.A. J. 8, 2172-2179.

Davidson, P. A. 2013 Turbulence in rotating, stratified and electrically conducting fluids. Cambridge University Press. 
Deloncle, A., Billant, P. \& Chomaz, J.-M. 2008 Nonlinear evolution of the zigzag instability in stratified fluids: a shortcut on the route to dissipation. J. Fluid Mech. 599, 229-239.

Deusebio, E., Boffetta, G., Lindborg, E. \& Musacchio, S. 2014 Dimensional transition in rotating turbulence. Phys. Rev. E 90 (2), 023005.

Gallaire, F. \& Rousset, F. 2006 Short-wave centrifugal instability in the vicinity of vanishing total vorticity streamlines. Phys. Fluids 18 (5), 058102.

Gallet, B. 2015 Exact two-dimensionalization of rapidly rotating large-reynolds-number flows. J. Fluid Mech. 783, 412-447.

Godeferd, F. S., Cambon, C. \& Leblanc, S. 2001 Zonal approach to centrifugal, elliptic and hyperbolic instabilities in stuart vortices with external rotation. J. Fluid Mech. 449, 1.

GodefERd, F. S. \& Moisy, F. 2015 Structure and dynamics of rotating turbulence: a review of recent experimental and numerical results. Applied Mechanics Reviews 67 (3).

Greenspan, H. P. 1968 The theory of rotating fluids. Cambridge University Press.

Julien, K. \& KNobloch, E. 2007 Reduced models for fluid flows with strong constraints. J. Math. Phys. 48 (6), 065405.

Julien, K., KNobloch, E. \& Werne, J. 1998 A new class of equations for rotationally constrained flows. Theor. Comput. Fluid Dyn. 11 (3-4), 251-261.

van Kan, A. \& Alexakis, A. 2020 Critical transition in fast-rotating turbulence within highly elongated domains. J. Fluid Mech. 899, A33.

Kerswell, R. R. 2002 Elliptical instability. Annu. Rev. Fluid Mech. 34, 83-113.

Kloosterziel, R. C. \& van Heisst, G. J. F 1991 An experimental study of unstable barotropic vortices in a rotating fluid. J. Fluid Mech. 223, 1-24.

Le Dizes, S. 2000 Three-dimensional instability of a multipolar vortex in a rotating flow. Phys. Fluids 12 (11), 2762-2774.

Leweke, T., Le Dizès, S. \& Williamson, C.H.K. 2016 Dynamics and instabilities of vortex pairs. Annu. Rev. Fluid Mech. 48, 507-541.

Moisy, F., Morize, C., Rabaud, M. \& Sommeria, J. 2011 Decay laws, anisotropy and cyclone-anticyclone asymmetry in decaying rotating turbulence. J. Fluid Mech. 666, 5.

Nazarenko, S. V. \& Scheкochinin, A. A. 2011 Critical balance in magnetohydrodynamic, rotating and stratified turbulence: towards a universal scaling conjecture. J. Fluid Mech. 677, 134-153.

Potylitsin, P. G. \& Peltier, W. R. 1999 Three-dimensional destabilization of Stuart vortices: the influence of rotation and ellipticity. J. Fluid Mech. 387, 205-226.

Seshasayanan, K. \& Gallet, B. 2020 Onset of three-dimensionality in rapidly rotating turbulent flows. $J$. Fluid Mech. 901.

SiPP, D. \& JACQUIN, L. 2000 Three-dimensional centrifugal-type instabilities of two-dimensional flows in rotating systems. Phys. Fluids 12 (7), 1740-1748.

SipP, D. \& JACQUIN, L. 2003 Widnall instabilities in vortex pairs. Phys. Fluids 15, 1861-1874.

SipP, D., JACQuin, L. \& Cossu, C. 2000 Self-adaptation and viscous selection in concentrated twodimensional dipoles. Phys. Fluids 12 (2), 245-248.

Sipp, D., Lauga, E. \& Jacquin, L. 1999 Vortices in rotating systems: centrifugal, elliptic and hyperbolic type instabilities. Phys. Fluids 11 (12), 3716-3728.

Sprague, M., Julien, K., Knobloch, E. \& Werne, J. 2006 Numerical simulation of an asymptotically reduced system for rotationally constrained convection. J. Fluid Mech. 551, 141. 